\title{
Impacto de uma Intervenção Psicoeducacional sobre o Bem-Estar Subjetivo de Cuidadores de Idosos com Doença de Alzheimer
}

\author{
Lais de Oliveira Lopes \\ Faculdade de Ciências Médicas da Universidade Estadual de Campinas, Campinas, Brasil \\ Meire Cachioni ${ }^{1}$ \\ Escola de Artes, Ciências e Humanidades da Universidade de São Paulo, São Paulo, Brasil \\ Faculdade de Ciências Médicas da Universidade Estadual de Campinas, Campinas, Brasil
}

\section{Resumo}

O objetivo deste estudo foi investigar o impacto de um programa psicoeducacional dirigido a cuidadores familiares de idosos com Doença de Alzheimer em relação às avaliações desses cuidadores sobre o seu bem-estar subjetivo. Cuidadores familiares de idosos $(n=21)$ foram entrevistados antes e após a intervenção, composta por quinze sessões. Os instrumentos aplicados foram: Escala de Satisfação Geral com a Vida; Escala de Satisfação com a Vida Referenciada a Domínios; Escala de Ânimo Positivo e Negativo; e, Escala de Depressão Geriátrica. Os cuidadores apresentaram melhora em satisfação geral com a vida e com o envolvimento social, e maior presença de afetos positivos. Ao comparar os valores correspondentes às diferenças de antes e após a intervenção das variáveis de bem-estar, observou-se que houve diferença significativa entre os cuidadores com renda superior a seis salários mínimos e os cuidadores de menor renda em relação à variável de satisfação com a capacidade mental, e entre os cuidadores que cuidavam há mais de doze meses e os que cuidavam há menos tempo quanto à satisfação geral com a vida. Tais resultados se diferenciaram entre grupos de cuidadores, de acordo com o sexo, gênero, graus de parentesco, renda, idade e tempo de cuidado.

Palavras-chave: Cuidadores, bem-estar, psicoeducação, doença de Alzheimer.

\section{Impact of a Psychoeducational Intervention on Subjective Well-Being of Caregivers of Patients with Alzheimer's Disease}

\begin{abstract}
The aim this study was to investigate the impact of a psychoeducational intervention for family caregivers of elderly with Alzheimer's disease in relation to assessments of carers about their subjective well-being. Family caregivers of elderly $(n=21)$ were interviewed before and after the intervention, consisting of fifteen sessions. Interviews were conducted with caregivers, using a sociodemographics form, Satisfaction with Life Scale, Satisfaction with Life Domains Scale, Positive Affect and Negative Affects Scale and Geriatric Depression Scale. The participants showed improvement in overall satisfaction with life and social involvement, and greater presence of positive affect. By comparing the values corresponding to the differences between before and after the intervention of the variables of well-being

Endereço para correspondência: Av. Arlindo Bettio, 1000, Edifício I1, sala 251E, Bairro Ermelino Matarazzo, São Paulo, SP, Brasil 03828-000. E-mail: laisgeronto@gmail.com e meirec@usp.br

Agradecimento ao apoio financeiro recebido pela Coordenação de Aperfeiçoamento de Pessoal de Nível Superior (CAPES) e ao Hospital Dia Geriátrico do Instituto de Psiquiatria da Faculdade de Medicina da Universidade de São Paulo por fornecer o espaço e os participantes para o estudo.
\end{abstract}


among gender, age, income, family relationship and care time, there was a significant difference among caregivers with incomes exceeding six salaries minimum and caregivers of lower income in relation to the variable of satisfaction with the mental capacity, and among caregivers who were caring for more than twelve months and who cared about the less time there was general satisfaction with life. These results differed between groups of caregivers, according to sex, gender, degree of kinship, income, age and time of care.

Keywords: Caregivers, well-being, psychoeducation, Alzheimer's disease

\section{Impacto de una Intervención Psicoeducativa en el Bienestar Subjetivo de los Cuidadores de Pacientes con Enfermedad de Alzheimer}

\section{Resumen}

El objetivo de este estudio fue investigarinvestigar el impacto de una intervención psicoeducativa dirigido a familiares cuidadores de personas con enfermedad de Alzheimer en relación con las evaluaciones de los cuidadores acerca de su bienestar subjetivo. Los cuidadores familiares $(n=21)$ fueron entrevistados antes y después de la intervención, que consta de quince sesiones. Los instrumentos utilizados fueron: Escala de Satisfacción General con la vida, Escala de Satisfacción con los ámbitos de la vida se hace referencia, Escala del Estado de Ánimo Positivo y Negativo, y Escala del Depresión Geriátrica. Los participantes mostraron una mejoría en la satisfacción general con la vida y la participación social, y una mayor presencia de afectos positivos. Hubo una diferencia significativa entre los cuidadores que ganaban más de seis del salario mínimo y los cuidadores que ganaban los salarios más bajos en relación a la variable de satisfacción con la capacidad mental, y entre los cuidadores que se cuidan a más de doce meses y que se cuidava por menos tiempo hay satisfacción general con la vida. Los resultados fueron diferentes entre los grupos de cuidadores, según el sexo, el género, el grado de parentesco, edad y tiempo de cuidado.

Palabras clave: Cuidadores, bienestar, psicoeducación, enfermedad de Alzheimer.

Cuidadores de idosos com Doença de Alzheimer (DA) desempenham um papel essencial, pois envolvem-se em praticamente todos os aspectos do cuidado, assumindo responsabilidades adicionais de maneira crescente. Ou seja, à medida que a doença progride, o paciente necessita cada vez mais de auxílios na realização das atividades instrumentais de vida diária (AIVD's). Nos estágios mais avançados é necessário que os cuidadores auxiliem efetivamente nas atividades básicas de vida diária (ABVD's), como tarefas de cuidado pessoal (Haley, 1997; Pinquart \& Sörensen, 2003). Dentre as tarefas realizadas pelos cuidadores de idosos com DA, administrar medicamentos, auxiliar no vestuário e preparar a alimentação, são as que mais requerem cuidado(Lemos, Gazzola, \& Ramos, 2006).

Nesse contexto, diversas são as dificuldades enfrentadas pelos cuidadores de idosos demenciados que comprometem fortemente o bem-estar subjetivo, como, por exemplo, a aceitação do diagnóstico, lidar com o estresse cada vez maior, administrar os conflitos dentro da família e ainda planejar o futuro (Abreu, Barros, \& Forlenza, 2005). A necessidade de cuidados ininterruptos, o difícil manejo das manifestações comportamentais, somados as vivências dos laços emocionais, tanto positivos como negativos vivenciados no convívio anterior à instalação da doença, provocam desgaste físico, mental e emocional. $\mathrm{O}$ cuidador de um paciente com DA é constantemente testado em sua capacidade de discernimento e adaptação à nova realidade, que exige alem de dedicação, responsabilidade, paciência e também abnegação. Esse papel gera sentimentos ambivalentes em relação ao idoso, testando seus limites psicológicos e sua postura de enfrentamento perante a vida (Luzardo, Gorini, \& Silva, 2006). 
Diversos estudos mostram o ônus gerado pelo ato de prestar cuidado. De acordo com Fortinsky, Tennen, Frank e Affleck (2007), os cuidadores estão em maior risco de morbidade psicológica e física e funcionamento social prejudicado, comprometendo, portanto, a capacidade de cuidar de um membro familiar idoso. Atualmente, sabe-se que as pessoas que prestam o cuidado possuem pior saúde emocional quando comparados aos não cuidadores (Aldwin, Yancura, \& Boeninger, 2007). Além de tais consequências, um cuidador de uma pessoa com demência enfrenta desafios como: falta de apoio informal (rede de suporte social informal, composta por membros familiares, amigos e vizinhos), falta de conhecimento de serviços formais e falta de preparação para a prestação de cuidados (Ducharme, Levesque, Lachance, Kergoat, \& Coulombe, 2011).

As evidências acumuladas sobre os impactos pessoais, sociais e de saúde do cuidador têm gerado estudos de intervenção que visam diminuir o estresse. A literatura aponta alguns tipos de intervenções utilizadas com cuidadores de idosos demenciados, dentre eles destacam-se: grupo de aconselhamento, grupo de apoio, psicoterapia, intervenção multicomponente e, intervenção psicoeducacional. Uma meta-análise feita por Sörensen, Pinquart e Duberstein (2002) de 78 estudos sobre intervenções em cuidadores, mostrou que, ao comparar com outras modalidades de intervenção (grupo de treinamento, gestão de caso/aconselhamento, grupo de apoio, entre outros), as intervenções psicoeducacionais tiveram efeitos mais consistentes na redução da sobrecarga e depressão, melhora do bem-estar subjetivo, aumento de habilidades e conhecimentos dos cuidadores e maior gerenciamento dos sintomas dos idosos que recebiam o cuidado.

Grupos psicoeducacionais podem colaborar para o manejo do cuidado e para o aumento do bem-estar dos cuidadores, à medida que transmitem conhecimentos que podem contribuir para a regulação das emoções. Constituem-se em espaços para trocas de experiências e sentimentos entre os membros (Lavoie et al., 2005). A psicoeducação é uma abordagem baseada em métodos experimentais e científicos, partindo do pressu- posto de que as cognições gerenciam as emoções e os comportamentos, ou seja, o componente cognitivo precede o componente afetivo, uma vez que ocorre transferência de informação e de descarga emocional (Brown, 2011).

Características relevantes dos grupos psicoeducacionais consistem em ser limitada no tempo, estruturada, diretiva, focada no presente e na resolução de problemas (Figueiredo Souza, Dell'Áglio, \& Argimon, 2009). Utiliza-se de técnicas como palestras, grupo de discussão, materiais escritos, uso de exercícios e recursos audiovisuais, que são gerenciados por um líder (Brown, 2011). Deste modo, esses grupos guardam semelhança com salas de aula, mas também com grupos de aconselhamento; contudo, a ênfase está na educação ou aprendizagem mais do que na autoconsciência ou autoentendimento, embora esses elementos estejam envolvidos.

De acordo com Chien et al. (2011), os grupos psicoeducacionais tendem a fornecer informações úteis, tais como habilidades de cuidados, formas de autoajustamento, conhecimento para lidar com questões legais, facilitando a busca de recursos disponíveis que podem reduzir a sobrecarga dos cuidadores mais rapidamente. Esses grupos não apenas fornecem informações práticas para o atendimento ao paciente, mas também focam no estado psicológico e emocional dos cuidadores, bem como contribuem para o estabelecimento de uma rede social de apoio.

Para Figueiredo et al. (2009), educar os pacientes e familiares torna-se fundamental, pois as informações sobre a patologia contribuem para que esses identifiquem os pensamentos e comportamentos distorcidos que geram aflição e sofrimento. Tal intervenção se torna cada vez mais relevante à medida que há carência de informações básicas ou treinamento formal adequado aos familiares e à comunidade para o manejo diário adequado dos indivíduos (Winefield \& Harvey, 2004).

Dessa forma, destaca-se a importância de grupos psicoeducacionais destinados aos familiares cuidadores, já que esses são um recurso importante no manejo do paciente e representam uma porta de entrada para a eficácia do tratamento e diminuição de gastos. O suporte faz-se 
necessário para permitir que os cuidadores informais sejam capazes de continuar o seu papel durante o maior tempo possível. O apoio deve incluir informações para ajudar a compreensão, habilidades para ajudar no manejo do cuidado e a oportunidade para permitir um envolvimento em outras atividades. Ressalta-se que proporcionar bem-estar para o cuidador pode contribuir na redução dos custos indiretos relacionados com os cuidadores e também postergar e/ou evitar institucionalização do paciente (World Health Organization, \& Alzheimer's Disease International, 2012).

Diante deste complexo contexto, o presente estudo teve como objetivo investigar o impacto de um programa psicoeducacional dirigido a cuidadores familiares de idosos com DA em relação às avaliações desses cuidadores sobre o seu bem-estar subjetivo, comparando tais medidas entre sexo, idade, renda, relação de parentesco e tempo de cuidado antes e após a intervenção.

\section{Método}

\section{População de Estudo}

A amostra correspondeu a 21 cuidadores informais participantes do Grupo de Intervenção Psicoeducacional para Cuidadores de Idosos com Doença de Alzheimer, que acompanham os pacientes com Doença de Alzheimer atendidos no Centro de Reabilitação e Hospital-Dia para Idosos do Instituto de Psiquiatria do Hospital das Clínicas da Faculdade de Medicina da Universidade de São Paulo.

Grupo de Intervenção Psicoeducacional para Cuidadores de Idosos com Doença de Alzheimer. Esse grupo teve o intuito de conhecer o processo da doença e compreender as diversas realidades do cuidado. Caracterizou-se como um espaço de aprendizagem mútua; de promoção de rede de apoio para o enfrentamento do processo da doença; e da busca de melhorias para o bem-estar subjetivo do cuidador. Foi desenvolvido a partir da perspectiva teórica definida por Brown (2011), a qual considera que o grupo psicoeducacional é uma ferramenta para educar e instruir os membros do grupo acerca de um particular assunto e fornecer informações de como enfrentar crises decorrentes da vida. Espera-se que a partir do conhecimento adquirido o sujeito gerencie suas emoções, ou seja, o componente cognitivo deve preceder o componente afetivo.

Em cada encontro foi abordado um tema alusivo ao processo da doença, e posteriormente foi aberto o diálogo livre para perguntas, reflexões, relatos e trocas de experiências sobre condutas para lidar com os problemas diários do ato de cuidar. Os temas abordados no grupo de intervenção psicoeducacional estão relacionados a cinco eixos de conhecimentos:

- Eixo 1. Cérebro e o processo demencial: Informações sobre a estrutura do cérebro e suas principais funções. Explicações detalhadas sobre as alterações que ocorrem no processo demencial. Apresentação dos principais processos demências e suas manifestações na estrutura cerebral.

- Eixo 2. Demência tipo Alzheimer: Detalhamento de todas as fases que compreendem a Doença de Alzheimer. Apresentação da evolução das descobertas científicas relativas ao processo da doença. Discussão sobre a importância de uma rede nacional de informações acerca do processo, tratamentos e redes de apoio.

- Eixo 3. Tratamento farmacológico e não farmacológico: Informações sobre os principais medicamentos disponíveis para o tratamento farmacológico e sua eficácia em cada fase do processo. Informações sobre a estimulação cognitiva realizada no programa e a especificidade da intervenção de cada área de atuação.

- Eixo 4. Alterações fisiológicas e comportamentais refletidas no cotidiano: Informações sobre as alterações ocorridas em cada fase e discussão sobre o manejo de cada demanda do cuidado. Pertinência das adaptações ambientais e ergonomia em cada domicílio. Discussão sobre ocupação do tempo livre em casa e no ambiente externo.

- Eixo 5. O cuidado: Discussão sobre as peculiaridades do cuidado formal e do informal. Manejo das emoções e sentimentos que envolvem o ato de cuidar. 


\section{Tabela 1}

Eixos e Conteúdos ministrados no Grupo de Intervenção Psicoeducacional para Cuidadores de Idosos com Doença de Alzheimer no Centro de Reabilitação e Hospital-Dia para Idosos do Instituto de Psiquiatria do Hospital das Clínicas da Faculdade de Medicina da Universidade de São Paulo

\begin{tabular}{|c|c|}
\hline Eixos & Conteúdo \\
\hline $\begin{array}{l}\text { 1. Cérebro } \\
\text { e o processo } \\
\text { demencial }\end{array}$ & $\begin{array}{l}\text {-O que é o cérebro? } \\
\text {-Senescência x Senilidade } \\
\text {-Relato de experiências } \\
\text {-O que é demência? } \\
\text {-Tipos mais frequentes de demências } \\
\text {-Relato de experiências }\end{array}$ \\
\hline $\begin{array}{l}\text { 2. Demência } \\
\text { tipo } \\
\text { Alzheimer }\end{array}$ & $\begin{array}{l}\text {-Doença de Alzheimer: Fatores causais e a importância da detecção precoce } \\
\text {-História da descoberta e principais avanços das pesquisas atuais } \\
\text { - Relato de experiências } \\
\text {-Doença de Alzheimer } \\
\text {-Alterações cerebrais e fases da doença (ênfase em inicial e moderada conforme a } \\
\text { composição do Grupo) } \\
\text {-Relato de experiências } \\
\text {-Doença de Alzheimer e a realidade mundial - apresentação de vídeos } \\
\text {-A importância da informação acerca da doença } \\
\text {-Relato de experiências }\end{array}$ \\
\hline $\begin{array}{l}\text { 3. Tratamento } \\
\text { farmacológico } \\
\text { e não farmacológico }\end{array}$ & $\begin{array}{l}\text {-Tratamento: Intervenção farmacológica e reabilitação cognitiva / Quais resultados } \\
\text { esperados nas fases inicial e moderada? } \\
\text {-A importância do trabalho da equipe multiprofissional } \\
\text {-Relato de experiências }\end{array}$ \\
\hline $\begin{array}{l}\text { 4. Alterações } \\
\text { fisiológicas } \\
\text { e comportamentais } \\
\text { refletidas } \\
\text { no cotidiano }\end{array}$ & $\begin{array}{l}\text {-O dia-a-dia do portador da Doença de Alzheimer (I) / O que podemos esperar nas } \\
\text { fases inicial e moderada? (AVDs e AVDIs - âmbito privado / ergonomia - disposição } \\
\text { da mobilidade e situações de risco; banheiro, vestuário \{cuidados pessoais\}, cozinha). } \\
\text {-Resolução de problemas para cada demanda } \\
\text {-Regularidade do estilo de vida nas fases } \\
\text {-Relato de experiências } \\
\text {-O dia-a-dia do portador da Doença de Alzheimer (II) / O que podemos esperar nas } \\
\text { fases inicial e moderada? (atividades/lazer, direção, administração financeira, situações } \\
\text { de risco externo) } \\
\text {-Resolução de problemas para cada demanda } \\
\text {-Regularidade do estilo de vida nas fases. } \\
\text {-Relato de experiências } \\
\text {-Aspectos nutricionais e a importância da atividade física } \\
\text {-Relato de experiências }\end{array}$ \\
\hline 5. O cuidado & $\begin{array}{l}\text {-Cinedebate: Trechos do longa metragem Íris } \\
\text {-Relato de experiências } \\
\text {-Cinedebate: Curta metragem Clarita } \\
\text {-Relato de experiências } \\
\text {-Cuidado familiar e cuidado formal } \\
\text {-Cuidado formal - Quais as demandas profissionais? } \\
\text { - Relato de experiências } \\
\text {-Reflexão sobre o cuidado } \\
\text {-Técnicas de gerenciamento do estresse } \\
\text {-Relato de experiências }\end{array}$ \\
\hline
\end{tabular}




\section{Instrumentos}

Ficha de Caracterização Sociodemográfica do Participante e Carecteristcas do Contexto do Cuidado. Tem o objetivo de levantar o perfil dos sujeitos envolvidos na pesquisa, no que se referem aos dados pessoais e de cuidado, tais como: idade, gênero, escolaridade, ocupação, e renda, relação de parentesco entre cuidador e pessoa cuidada, tempo de cuidado, divisão da tarefa de cuidado e co-residência entre cuidador e idoso cuidado.

Escala de Satisfação Geral com a Vida (Neri, 2002). Instrumento para medida de bem-estar subjetivo indicado por satisfação geral com a vida, através da figura de uma escada de dez degraus simbolizando uma escala de dez pontos entre a pior vida (1 ponto) e a melhor vida (10 pontos).

Escala de Satisfação Geral com a Vida Referência a Domínios (Neri, 2002). A satisfação referenciada a domínios é avaliada por meio de um inventário de quatro itens com cinco opções de resposta variando de "pouquíssimo a muitíssimo satisfeito", avaliando os seguintes domínios: saúde, capacidade física, capacidade mental e envolvimento social. Essa escala tem uma validade interna superior a 0,80 e uma correlação com a medida de satisfação global superior a $90 \%$.

Escala de Afetos Positivos e Negativos (Siqueira, Martins, \& Moura, 1999). Essa escala tem como objetivo de avaliar a intensidade dos estados afetivos no cotidiano. Ela se constitui de duas sub-escalas, uma com oito itens referentes ao ânimo negativo (irritado, desmotivado, angustiado, deprimido, chateado, nervoso, triste, desanimado), e a outra com seis itens que correspondem ao ânimo positivo (feliz, alegre, animado, bem, satisfeito, contente), com índices de confiabilidade (alfa de Cronbach) de 0,88 e 0,87, respectivamente, com um alfa para a escala geral de 0,91 . A intensidade dos sentimentos é expressa através de uma escala que varia de 1 (pouco) a 5 (extremamente).

Escala de Depressão Geriátrica (GDS; Sheikh \& Yesavage, 1986; Almeida \& Almeida, 1999). A GDS é composta por 15 itens que mais fortemente se correlacionam com o diagnóstico de depressão. Esses itens, em conjunto, mostram boa acurácia diagnóstica, com sensibilidade, especificidade e confiabilidade adequadas. Essa versão reduzida é bastante atraente para rastreamento dos transtornos do humor em ambulatórios gerais, assim como em outros ambientes não-especializados, pois o tempo necessário para a sua administração é menor. O ponto de corte adotado é de maior ou igual a seis itens para rastreio de sintomas depressivos.

\section{Procedimentos}

Os sujeitos foram abordados pela pesquisadora principal e pelas colaboradoras que são alunas do curso de Gerontologia da Escola de Artes, Ciências e Humanidades, da Universidade de São Paulo (USP). As auxiliares foram previamente treinadas para aplicação dos instrumentos.

No primeiro dia de intervenção, todos os cuidadores familiares que estavam presentes responderam a um protocolo de instrumentos previamente validados. Ao término das 15 sessões foram entrevistados apenas os cuidadores que tiveram presença assídua no "Grupo de Intervenção Psicoeducacional para Cuidadores de Idosos com Doença de Alzheimer", com presença mínima em 13 encontros, para evitar possível viés nos resultados da pesquisa.

\section{Critério de Exclusão}

Foram excluídos os cuidadores que participavam de qualquer outro tipo de intervenção, como grupos de apoio, de aconselhamento e terapêuticos destinados à cuidadores de idosos. Os que frequentaram menos de 13 encontros, também foram excluídos da presente pesquisa.

\section{Análise dos Dados}

As informações obtidas mediante a aplicação dos instrumentos foram submetidas à análise estatística descritiva e a testes não paramétricos. Por seu intermédio obteve-se dados de frequências absolutas e relativas. Para comparação das variáveis categóricas foram utilizados os testes Qui-Quadrado ou exato de Fisher (para valores esperados menores que 5), o teste de Mann-Whitney para comparação das variáveis numéricas entre dois grupos, e o teste de Kruskal-Wallis 
para comparação das variáveis numéricas entre três ou mais grupos. Para analisar a relação entre as variáveis numéricas foi utilizado o coeficiente de correlação de Spearman, devido ao tamanho da amostra e à ausência de distribuição Normal. O nível de significância adotado para os testes estatísticos foi de $5 \%$, ou seja, $P<0,05$.

Os dados da Escala de Afetos Positivos e Afetos Negativos foram analisados a partir da média dos itens positivos (escore varia de 1 a 5), e da média dos itens negativos, cujo escore também varia de 1 a 5 pontos. Depois, foi feito a diferença entre o escore de afetos positivos (AP) e o escore de afetos negativos (AN), que corresponde ao valor delta, ou seja, Delta $=\mathrm{AP}-$ -AN. Dessa forma, o delta refere-se ao escore médio da Escala de Afetos Positivos e Negativos. Quando esse escore apresenta o valor negativo, deve-se considerar que houve mais afetos negativos que afetos positivos.

As opções de respostas dos itens da Escala de Satisfação geral com a Vida, (de "pouquíssimo a muitíssimo satisfeito") foram consideradas como uma variável escalar ordinal, assumindo a pontuação de 1 a 5 . Dessa forma foi possível obter os valores de média, desvio padrão e mediana, para que pudessem ser feitas as comparações e relações com as demais variáveis. Quanto mais próximo de um, menor é a satisfação, e quanto mais próximo de cinco, maior a satisfação.

Os valores correspondentes às diferenças entre a avaliação do pré e do pós-teste foram realizadas da seguinte forma: para cada variável foi feito a diferença do escore entre os tempos pré e pós-teste (valor do pós-teste - valor do pré-teste). Se o resultado dessa subtração for negativo, o escore caiu entre um tempo e o outro, e se for positivo, houve um aumento do valor. Por exemplo, se o valor da diferença entre e pré e pós-teste para a variável satisfação geral com a vida for positivo, deve-se considerar que após a intervenção houve aumento da satisfação geral com a vida. Na Escala de Depressão Geriátrica, quanto maior o escore, maior o número de repostas referentes aos sintomas depressivos. Assim para essa escala, quando a diferença dos valores entre o pré e o pós-teste for positiva, deve-se interpretar que houve aumento dos sintomas de depressão após a participação na intervenção.

\section{Resultados}

As características sociodemográficas da amostra estão apresentadas na Tabela 2. Aproximadamente $70 \%$ são cuidadores do sexo feminino, com idade média de 59,6 anos. A situação

Tabela 2

Descrição Sociodemográfica e Características da Situação de Cuidado da Amostra Composta por 21 Cuidadores Familiares Participantes de uma Intervenção Psicoeducacional

\begin{tabular}{lcc}
\hline \multicolumn{1}{c}{ Variáveis } & N & $\%$ \\
\hline Sexo & & \\
Masculino & 7 & 33,3 \\
Feminino & 14 & 66,7
\end{tabular}

Faixa Etária

Menor que 60 anos

$8 \quad 38,1$

Maior ou igual a 60 anos

$13 \quad 61,9$

Escolaridade

E. Fundamental

$4 \quad 19,1$

E. Médio

$7 \quad 33,3$

E. Superior

$10 \quad 47,6$

Renda familiar

Menor ou igual a $5 \mathrm{SM}$

1470,0

Maior ou igual a $6 \mathrm{SM}$

630,0

Situação Trabalhista

Ativo

$8 \quad 38,0$

Não ativo

$13 \quad 61,9$

Relação de parentesco

Cônjuge

1257,1

Filho

4

Outros (irmão, neto, sobrinho)

$5 \quad 23,8$

Divisão da terefa de cuidar

Sim

$7 \quad 33,3$

Não

$14 \quad 66,7$

Divisão da moradia com o paciente

Sim

$17 \quad 80,9$

Não

$4 \quad 19,0$ 
trabalhista dos cuidadores foi dividida em ativos e não-ativos, os primeiros correspondem aos cuidadores empregados, aos que são aposentados mas trabalham e às donas de casa, os não-ativos são os desempregados, aposentados e pensionistas. A média do tempo de cuidado oferecido ao familiar foi de 27,3 meses. Em relação ao grau de parentesco, em torno de $57 \%$ eram os cônjuges que forneciam o cuidado, seguido de outros familiares como irmãos, netos e sobrinhos, e depois, filhos. Todos os idosos cuidados tinham o diagnóstico médico de Doença de Alzheimer, sendo que esses estavam na fase leve ou modera- da da doença, correspondendo a 52,4\% e 47,6\%, respectivamente.

Conforme a Tabela 3, os resultados mostraram que os cuidadores de idosos com Doença de Alzheimer que participaram da intervenção psicoeducacional tiveram um aumento não significativo da satisfação geral com a vida, da satisfação com o envolvimento social e da presença de afetos positivos. Houve leve diminuição da satisfação com a saúde, com a capacidade física e aumento dos sintomas depressivos. Não houve diferença entre o pré e o pós-teste em relação à satisfação com a capacidade mental.

Tabela 3

Análise Descritiva da Comparação entre os Resultados do Pré e Pós-Teste Avaliados na Intervenção Psicoeducacional em Relação às Variáveis de Bem-Estar Investigadas nos Cuidadores Familiares de Idosos com Doença de Alzheimer

\begin{tabular}{lcccccc}
\hline \multirow{2}{*}{\multicolumn{1}{c}{ Variável }} & \multicolumn{2}{c}{ Média } & \multicolumn{2}{c}{ Desvio-padrão } & \multicolumn{2}{c}{ Mediana } \\
\cline { 2 - 7 } & Pré-teste & Pós-teste & Pré-teste & Pós-teste & Pré-teste & Pós-teste \\
\hline Satisfação geral com a vida & 6,9 & 6,95 & 1,89 & 1,83 & 8 & 8 \\
Satisfação referenciada a domínios: & & & & & & \\
Saúde & 3,48 & 3,33 & 0,93 & 0,66 & 4 & 3 \\
Capacidade física & 3,67 & 3,33 & 0,97 & 1,06 & 4 & 3 \\
Capacidade mental & 3,81 & 3,81 & 1,03 & 0,98 & 4 & 4 \\
Envolvimento pessoal & 3,19 & 3,38 & 1,08 & 1,12 & 3 & 4 \\
Sintomas depressivos (GDS) & 3,62 & 4,24 & 2,65 & 3,33 & 3 & 4 \\
Afeto Negativo & 2,24 & 2,13 & 0,91 & 0,76 & 2 & 2 \\
Afeto Positivo & 3,32 & 3,47 & 0,78 & 0,83 & 3,5 & 3,5 \\
Delta Afeto Positivo e Afeto Negativo & 1,08 & 1,34 & 1,58 & 1,28 & 1,5 & 1,54 \\
\hline
\end{tabular}

Os valores correpondentes às diferenças entre o pré e pós-teste das variáveis de bem-estar entre grupos divididos em sexo, idade, renda, relação de parentesco e tempo de cuidado mostraram que houve diferença significativamente estatística entre os grupos que cuidavam há mais de 12 meses e os que cuidavam há menos tempo em relação à variável de satisfação geral com a vida, e diferença significativa entre os grupos de diferentes rendas para a variável de satisfação com a capacidade mental (Tabela 4).

Após a participação no grupo, as mulheres cuidadoras apresentaram discreta diminuição da satisfação geral com a vida e com a capacidade física e aumento do escore na escala de sintomas depressivos. Por outro lado, houve aumento no escore de satisfação com a capacidade mental e aumento de afetos positivos. Os homens tiveram aumento da satisfação geral com a vida e com o envolvimento social, aumento de afetos positivos, e diminuição na pontuação de itens na escala de depressão geriátrica após a participação no grupo. Eles apresentaram redução na satisfação com a saúde, com a capacidade física e com a capacidade mental.

Observou-se maior frequência de cuidadores cônjuges com idade acima de 60 anos e cuidadores filhos com idade inferior a 60 anos ( $p=0,013$, no Teste Exato de Fisher). Depois da participação na intervenção, cuidadores com 
Tabela 4

Valores de Média, Mínimo, Mediana e Máximo Correspondentes às Diferenças entre Pré e Pós-Teste das Variáveis de Bem-Estar entre Sexo, Idade, Renda e Tempo de Cuidado. Valor p Referente ao Teste de Mann-Whitney para Comparação de Valores entre as Variáveis Numéricas (dois grupos)

\begin{tabular}{|c|c|c|c|c|c|c|c|c|c|}
\hline & Média $(D P)$ & Mínimo & Mediana & Máximo & Média $(D P)$ & Mínimo & Mediana & Máximo & Valor- $p$ \\
\hline Varlaveis de Bem-Estar & \multicolumn{4}{|c|}{ Sexo Feminino } & \multicolumn{4}{|c|}{ Sexo Masculino } & \\
\hline Satisfação geral com a vida & $-0,21(1,37)$ & -3 & $-0,5$ & 2 & $0,57(0,98)$ & 0 & 0 & 2 & 0,128 \\
\hline \multicolumn{10}{|c|}{ Satisfação referenciada a domínios: } \\
\hline Saúde & $0(0,88)$ & -2 & 0 & 2 & $-0,43(0,53)$ & -1 & 0 & 0 & 0,141 \\
\hline Capacidade física & $-0,36(0,93)$ & -2 & $-0,5$ & 1 & $-0,29(0,95)$ & -2 & 0 & 1 & 0,753 \\
\hline Capacidade mental & $0,14(0,95)$ & -1 & 0 & 2 & $-0,29(0,49)$ & -1 & 0 & 0 & 0,316 \\
\hline Envolvimento pessoal & $0(1,18)$ & -2 & 0 & 3 & $0,57(1,13)$ & -1 & 0 & 2 & 0,266 \\
\hline Sintomas depressivos (GDS) & $1(2,22)$ & -2 & 0,5 & 6 & $-0,14(2,04)$ & -3 & 0 & 3 & 0,327 \\
\hline \multirow[t]{2}{*}{ Delta APN } & $0,18(1,12)$ & $-1,71$ & 0,08 & 1,96 & $0,44(2,00)$ & $-2,83$ & $-0,33$ & 2,67 & 0,881 \\
\hline & \multicolumn{4}{|c|}{ Idade abaixo de 60 anos } & \multicolumn{4}{|c|}{ Idade igual ou acima de 60 anos } & \\
\hline Satisfação geral com a vida & $-0,38(1,41)$ & -3 & 0 & 2 & $0,31(1,18)$ & -1 & 0 & 2 & 0,344 \\
\hline \multicolumn{10}{|c|}{ Satisfação referenciada a domínios: } \\
\hline Saúde & $0,13(0,83)$ & -1 & 0 & 2 & $-0,31(0,75)$ & -2 & 0 & 1 & 0,319 \\
\hline Capacidade física & $-0,25(1,04)$ & -2 & 0 & 1 & $-0,38(0,87)$ & -2 & 0 & 1 & 0,703 \\
\hline Capacidade mental & $0(0,53)$ & -1 & 0 & 1 & $0(1,00)$ & -1 & 0 & 2 & 0,815 \\
\hline Envolvimento pessoal & $0,38(1,19)$ & -1 & 0 & 2 & $0,08(1,19)$ & -2 & 0 & 3 & 0,616 \\
\hline Sintomas depressivos (GDS) & $-0,13(1,64)$ & -2 & $-0,5$ & 2 & $1,08(2,4)$ & -3 & 1 & 6 & 0,242 \\
\hline \multirow[t]{2}{*}{ Delta APN } & $0,7(1,5)$ & $-1,71$ & 0,69 & 2,67 & $0(1,32)$ & $-2,83$ & $-0,04$ & 2,29 & 0,538 \\
\hline & \multicolumn{4}{|c|}{ Renda menor ou igual a 5 salários mínimos } & \multicolumn{5}{|c|}{ Renda maior ou igual a 6 salários mínimos } \\
\hline Satisfação geral com a vida & $0,07(1,49)$ & -3 & 0 & 2 & $-0,17(0,75)$ & -1 & 0 & 1 & 0,761 \\
\hline \multicolumn{10}{|c|}{ Satisfação referenciada a domínios: } \\
\hline Saúde & $-0,07(0,83)$ & -2 & 0 & 2 & $-0,33(0,82)$ & -1 & $-0,5$ & 1 & 0,355 \\
\hline Capacidade física & $-0,14(0,95)$ & -2 & 0 & 1 & $-0,5(0,55)$ & -1 & $-0,5$ & 0 & 0,358 \\
\hline Capacidade mental & $0,29(0,83)$ & -1 & 0 & 2 & $-0,67(0,52)$ & -1 & -1 & 0 & $0,017^{*}$ \\
\hline Envolvimento pessoal & $0,29(0,99)$ & -1 & 0 & 2 & $0(1,67)$ & -2 & 0 & 3 & 0,46 \\
\hline Sintomas depressivos (GDS) & $0,21(2,04)$ & -3 & 0 & 4 & $1,67(2,5)$ & -1 & 1 & 6 & 0,243 \\
\hline \multirow[t]{2}{*}{ Delta APN } & $0,56(1,48)$ & $-1,71$ & 0,56 & 2,67 & $-0,37(1,30)$ & $-0,37$ & $-0,21$ & 0,88 & 0,283 \\
\hline & \multicolumn{4}{|c|}{$\begin{array}{l}\text { Tempo de cuidado menor ou igual a } 12 \\
\text { meses }\end{array}$} & \multicolumn{5}{|c|}{ Tempo de cuidado maior que 12 meses } \\
\hline Satisfação geral com a vida & $-0,6(1,07)$ & -3 & $-0,5$ & 1 & $0,64(1,21)$ & -1 & 0 & 2 & $0,036^{*}$ \\
\hline \multicolumn{10}{|c|}{ Satisfação referenciada a domínios: } \\
\hline Saúde & $-0,1(0,32)$ & -1 & 0 & 0 & $-0,18(1,08)$ & -2 & 0 & 2 & 0,613 \\
\hline Capacidade física & $-0,1(0,99)$ & -2 & 0 & 1 & $-0,55(0,82)$ & -2 & -1 & 1 & 0,236 \\
\hline Capacidade mental & $-0,1(0,88)$ & -1 & 0 & 2 & $-0,09(0,83)$ & -1 & 0 & 1 & 0,677 \\
\hline Envolvimento pessoal & $0,1(0,88)$ & -1 & 0 & 2 & $0,27(1,42)$ & -2 & 0 & 4 & 0,793 \\
\hline Sintomas depressivos (GDS) & $0,1(1,87)$ & -2 & 0 & 3 & $1,00(2,45)$ & -3 & 1 & 6 & 0,393 \\
\hline Delta APN & $-0,19(1,5)$ & $-2,83$ & $-0,15$ & 2,13 & $0,68(1,29)$ & $-1,42$ & 0,5 & 2,67 & 0,231 \\
\hline
\end{tabular}

Notas. *nível de significância adotado: $p<0,05$. DP: Desvio-Padrão. Delta APN: Delta de afetos positivos e afetos negativos.

idade inferior a 60 anos tiveram menor satisfação geral com a vida e com a capacidade física. Porém, apresentaram melhora na satisfação com a saúde, com o envolvimento social, diminuição no escore da escala de sintomas depressivos e maior presença de afetos positivos. Os cuidadores idosos, maior de 60 anos, apresentaram aumento da satisfação geral com a vida e com o 
envolvimento social. Diminuiu a satisfação com a saúde e com a capacidade física e aumentaram o escore na escala de sintomas depressivos após a participação na intervenção.

Cuidadores com renda inferior a cinco salários mínimos tiveram aumento da satisfação geral com a vida, com a capacidade mental, com o envolvimento social e aumento de afetos positivos. Apresentaram leve piora na satisfação com a saúde e com a capacidade física. Os cuidadores com renda acima de seis salários mínimos relataram maior insatisfação que os cuidadores de renda abaixo desse valor: piora da satisfação geral com a vida, com a saúde, com a capacidade mental (estatisticamente significativo, quando comparado com o grupo de menor renda), e maior presença de afetos negativos e sintomas depressivos após a participação no grupo. Esse grupo não apresentou diferença entre o pré e pós-teste para a variável satisfação com a capacidade mental.

Os cuidadores que cuidavam há menos de 12 meses tiveram diminuição da satisfação geral com a vida, com a saúde, com a capacidade física, aumento no escore da escala de depres- são e maior presença de afetos negativos após a participação na intervenção. Houve aumento da satisfação com a capacidade mental e com o envolvimento social.

Já os cuidadores que cuidavam há mais de 12 meses aumentaram a satisfação geral com a vida (comparando com os cuidadores que cuidam há menos tempo, o valor de $p$ foi de 0,036 ), aumento da satisfação com o envolvimento social e da presença de afetos positivos. A satisfação com a saúde, com a capacidade física e com a capacidade mental, diminuíram após a participação no grupo.

No teste exato de Fisher verificou-se a diferença estatisticamente significativa entre relação de parentesco e divisão da tarefa de cuidar $(p=0,010)$. Foi mostrado que $91,6 \%$ dos cuidadores cônjuges não dividiam a tarefa de cuidado, já os cuidadores que possuem outras relações de parentesco (irmãos, sobrinhos e netos) foram os que mais dividiam essa tarefa $(80 \%)$.

Os resultados referentes à comparação das variáveis de bem-estar entre cônjuges, filhos e outros graus de parentesco revelaram que não há diferença estatisticamente significativa entre esses grupos (Tabela 5).

\section{Tabela 5}

Valores de Média, Mínimo, Mediana e Máximo Correspondentes às Diferenças entre Pré e Pós-Teste das Variáveis de Bem-Estar em Relação ao Grau de Parentesco dos Cuidadores com seus Familiares com Doença de Alzheimer. Valor p Referente ao Teste de Kruskal-Wallis para Comparação das Variáveis entre os Três Grupos.

\begin{tabular}{|c|c|c|c|c|c|c|c|c|c|c|c|c|c|}
\hline \multirow{2}{*}{$\begin{array}{l}\text { Valores das diferenças } \\
\text { entre pré e pós-teste }\end{array}$} & $\begin{array}{c}\text { Média } \\
(D P)\end{array}$ & Mín. & Mediana & Máx. & $\begin{array}{l}\text { Média } \\
(D P)\end{array}$ & Mín. & Mediana & Máx. & $\begin{array}{c}\text { Média } \\
(D P)\end{array}$ & Mín. & Mediana & Máx. & $p$ \\
\hline & \multicolumn{4}{|c|}{ Cônjuge } & \multicolumn{4}{|c|}{ Filho(a) } & \multicolumn{4}{|c|}{ Outros } & \\
\hline Satisfação geral com a vida & $\begin{array}{c}0 \\
(1,41)\end{array}$ & -3 & 0 & 2 & $\begin{array}{c}0,25 \\
(1,26)\end{array}$ & -1 & 0 & 2 & $\begin{array}{c}0 \\
(1,22)\end{array}$ & -1 & 0 & 2 & 0,935 \\
\hline \multicolumn{14}{|l|}{$\begin{array}{l}\text { Satisfação referenciada } \\
\text { a domínios: }\end{array}$} \\
\hline Saúde & $\begin{array}{l}-0,42 \\
(0,79)\end{array}$ & -2 & 0 & 1 & $\begin{array}{l}0,5 \\
(1)\end{array}$ & 0 & 0 & 2 & $\begin{array}{c}0 \\
(0)\end{array}$ & 0 & 0 & 0 & 0,144 \\
\hline Capacidade física & $\begin{array}{l}-0,58 \\
(0,79)\end{array}$ & -2 & -1 & 1 & $\begin{array}{c}-0,5 \\
(1,29)\end{array}$ & -2 & $-0,5$ & 1 & $\begin{array}{c}0,4 \\
(0,55)\end{array}$ & 0 & 0 & 1 & 0,1 \\
\hline Capacidade mental & $\begin{array}{l}-0,25 \\
(0,87)\end{array}$ & -1 & $-0,5$ & 1 & $\begin{array}{l}0,25 \\
(0,5)\end{array}$ & 0 & 0 & 1 & $\begin{array}{c}0,4 \\
(0,89)\end{array}$ & 0 & 0 & 2 & 0,275 \\
\hline Envolvimento pessoal & $\begin{array}{c}0,17 \\
(1,40)\end{array}$ & -2 & 0 & 3 & $\begin{array}{c}0 \\
(0,82)\end{array}$ & -1 & 0 & 1 & $\begin{array}{c}0,4 \\
(0,89)\end{array}$ & 0 & 0 & 2 & 0,846 \\
\hline Sintomas depressivos (GDS) & $\begin{array}{c}1,42 \\
(2,07)\end{array}$ & -1 & 1 & 6 & $\begin{array}{l}-0,25 \\
(1,71)\end{array}$ & -2 & $-0,5$ & 2 & $\begin{array}{c}-0,6 \\
(2,30)\end{array}$ & -3 & -1 & 3 & 0,128 \\
\hline $\begin{array}{l}\text { Delta Afeto Positivo } \\
\text { e Afeto Negativo }\end{array}$ & $\begin{array}{l}-0,34 \\
(1,17) \\
\end{array}$ & $-2,83$ & $-0,15$ & 1,42 & $\begin{array}{c}1,47 \\
(1,28) \\
\end{array}$ & $-0,33$ & 1,77 & 2,67 & $\begin{array}{c}0,76 \\
(1,53) \\
\end{array}$ & $-1,29$ & 0,88 & 2,29 & 0,0 \\
\hline
\end{tabular}

Nota. DP: Desvio-Padrão. 
Os cuidadores cônjuges apresentaram discreta piora da satisfação com a saúde, capacidade física e capacidade mental, aumento do respostas na escala de depressão geriátrica e maior presença de afetos negativos após a participação no grupo. O benefício foi em relação ao aumento da satisfação com o envolvimento social.

Já os filhos tiveram aumento da satisfação geral com a vida, com a saúde e com a capacidade mental, diminuição do escore na escala referente aos sintomas depressivos e aumento dos afetos positivos, após a participação na intervenção. Em contrapartida, houve diminuição da satisfação com a capacidade fisica. Na categoria "outros", observou-se que a intervenção teve impacto positivo em praticamente todas variáveis de bem-estar estudadas.

\section{Discussão}

Os resultados sobre o aumento do bem-estar subjetivo, avaliados pela escala de satisfação geral com a vida, satisfação referenciada a domínios, afetos positivos e afetos negativos e sintomas depressivos foram discretos, o que podem ser justificados pelo tamanho da amostra e pela variabilidade de características do grupo de cuidadores em relação aos diferentes graus de parentesco, gênero, idade e tempo dedicado ao cuidado.

A intervenção psicoeducacional investigada apresentou um impacto positivo no bem-estar subjetivo em relação às variáveis de satisfação geral com a vida, satisfação com o envolvimento social e equilíbrio entre afetos positivos e afetos negativos. Contudo, esta intervenção não contribuiu para a diminuição do escore na escala de depressão geriátrica, para o aumento da satisfação com a saúde e da satisfação com a capacidade física. Embora os valores em relação às variáveis de satisfação com a saúde e com a capacidade física tenham sido discretamente reduzidos e o escore de sintomas de depressão levemente aumentado no pós-teste. Não houve diferença entre antes e depois da intervenção para a variável de satisfação com a capacidade mental.

Tais resultados sugerem que a intervenção psicoeducacional, baseada no modelo proposto por Brown (2011), o qual considera que a partir do conhecimento adquirido acerca da doença e sobre as maneiras de enfrentamento da situação, o indivíduo maneje suas emoções, pode não ter sido totalmente eficaz para o aumento do bem-estar em relação à todas as variáveis estudadas. É importante destacar que na intervenção psicoeducacional desenvolvida neste estudo, não foram ensinadas estratégias de regulação emocional, pois o foco primordial consistiu-se na transmissão de conhecimentos sobre o processo da doença, e nas possíveis estratégias de lidar com as demandas diárias.

Observou-se que a média de escore não atingiu o ponto de corte para sintomas depressivos nem antes e nem após a intervenção, contudo, aumentaram a frequência de respostas relacionadas aos itens de sintomas de depressão no pós-teste. Embora haja evidências científicas de que determinados modelos de intervenções psicoeducacionais influenciam positivamente na redução dos sintomas depressivos, alguns autores discutem que as intervenções destinadas aos cuidadores de idosos com demência não impedem o curso de progressão da doença e não aliviam os aspectos dolorosos inerentes à situação (Aneshensel, Pearlin, Mulan, Zarit, \& Whitlach, 1995). Os cuidadores são confrontados frequêntemente com suas emoções angustiantes geradas pela perda e deterioração diária do seu familiar (Ducharme, Levesque, Giroux, \& Lachance, 2005). Para Gottlieb, Thompson e Bourgeois (2003), é esperado que os cuidadores sintam-se mais deprimidos, mesmo quando participantes de intervenções, devido às demandas estressantes, aos desafios envolvidos no cuidado de um parente com demência, e às ameaças futuras que acompanham o conhecimento da natureza do curso de deterioração da doença. De acordo com Sörensen et al. (2002), os níveis de prestação de cuidados, medidos pelo tempo diário dedicado ao cuidado podem ser relativamente elevados, e as intervenções para cuidadores podem não contribuir para a diminuição dos sintomas depressivos.

Em relação às variáveis de satisfação com a saúde e satisfação com a capacidade física, itens da escala de satisfação com a vida referenciada a domínios, a intervenção psicoeducacional não apresentou efeito positivo, provavelmente, 
porque os cuidadores eram predominantemente idosos $(62 \%)$ e não dividiam as tarefas de cuidado com alguém (67\%). A literatura mostra que os cuidadores idosos são menos satisfeitos com a saúde e com a capacidade física e que os cuidadores únicos sentem-se sobrecarregados fisicamente e têm saúde debilitada (Dahlberg, Demack, \& Bambra, 2007; Pimenta, Costa, Gonçalvez, \& Alvarez, 2009).

Contudo, parte desses resultados foi condizente com alguns estudos da literatura. No estudo de Arritxabal et al. (2011) em que foi avaliado uma intervenção psicoeducacional de dez sessões em 52 cuidadores, os resultados mostraram que após a intervenção aumentaram os afetos positivos e o bem-estar (avaliado pela satisfação com a vida). Em outros estudos, a intervenção psicoeducacional contribuiu para uma redução significativa em sentimentos como angústia, ira, medo, hostilidade e raiva (Andrade, 2009; Gallagher-Thompson \& DeVries, 1994; Hosaka \& Sugyama, 2003). A redução de afetos negativos após a intervenção provavelmente se deu devido às informações fornecidas sobre o gerenciamento adequado dos sintomas e dos problemas de comportamento dos idosos e pelo apoio emocional oferecido que podem ter contribuído para a melhora do estado de ânimo dos cuidadores e para maior confiança no oferecimento do cuidado ao seu parente.

À medida que se compreende o processo da doença, espera-se, que o cuidador se apodere de tal conhecimento recebido para lidar melhor com a situação, tanto no aspecto prático das demandas diárias, como no controle emocional (Brown, 2011; Figueiredo et al., 2009). É esperado que o cuidador considere essa condição como uma realidade que independe da vontade do idoso demenciado, de forma que não o culpabilize pelos comportamentos inadequados, diminuindo, então, os afetos negativos pelo idoso cuidado.

Destaca-se as diferenças dos resultados das variáveis estudadas entre os sexos masculino e feminino, idade abaixo e acima de 60 anos, diferentes rendas e relações de parentesco e tempo de cuidado dedicado ao idoso com DA. Os cuidadores que apresentaram benefícios em mais variáveis de bem-estar, com a participação no grupo psicoeducacional, foram os cuidadores do sexo masculino, com idade inferior a 60 anos, os filhos e outros parentes (como irmãos, sobrinhos e netos), os com renda inferior a cinco salários mínimos e os que cuidavam há mais de 12 meses.

Em relação ao sexo, os homens cuidadores tiveram aumento da satisfação geral com a vida e com o envolvimento social, aumento dos afetos positivos e diminuição do escore na escala de sintomas depressivos. Já, as mulheres tiveram aumento da satisfação com a capacidade mental e aumento dos afetos positivos após a intervenção.

Em uma meta-análise de 127 estudos de intervenções para cuidadores de idosos com demência, realizada por Pinquart e Sörensen (2006a) observou-se que nos estudos avaliados em que havia uma maior proporção de mulheres, os efeitos da intervenção sobre os sintomas depressivos foram mais significativos, enquanto que os efeitos sobre o bem-estar subjetivo foram mais fracos. Contudo no presente estudo, as mulheres tiveram aumento dos sintomas depressivos e os homens diminuição desses sintomas após a participação na intervenção. Esses resultados podem ser justificados pelo fato de que mulheres cuidadoras são mais deprimidas do que os cuidadores do sexo masculino e porque homens têm menos probabilidade de admitir sentimentos negativos, independentemente da intervenção (Pinquart \& Sörensen, 2006b). De acordo com Bookwala e Schulz (2000), os homens cuidadores prestam frequentemente menos cuidado do que as mulheres, e isso é uma explicação plausível para que eles também relatem menos sintomas depressivos do que as mulheres cuidadoras. Sem contar que, eles podem ser mais propensos a responder à intervenção com sentimentos positivos, em vez de culpa, e a também usarem intervenções como suporte para a decisão de institucionalizar o idoso dementado (Pinquart \& Sörensen, 2006a).

Diferentemente dos homens cuidadores, as mulheres não apresentaram aumento da satisfação com o envolvimento social, porque provavelmente, elas assumem outros papéis além de cuidadoras e provêm mais horas de cuidado do que os homens cuidadores (Pinquart \& Sörensen, 2006b). 
Cuidadores mais jovens tiveram aumento da satisfação com a saúde e com o envolvimento social, diminuição dos sintomas depressivos e aumento de afetos positivos. Enquanto que os cuidadores idosos tiveram aumento apenas da satisfação geral com a vida e com o envolvimento social.

Sabe-se que idosos sofrem mais para prover o cuidado (Dahlberg et al., 2007), pois, geralmente, têm a saúde debilitada e estão vivenciando as consequências do próprio envelhecimento, no que diz respeito ao funcionamento dos sistemas imunológico e neuroendócrino, responsáveis pelos processos reguladores do organismo (Neri, 2010). Além disso, eles parecem ser menos capazes de responder positivamente aos comportamentos inadequados de seu parente (Bookwala, 2009). Nesse estudo, um dado que deve ser levado em conta é que os cuidadores com idade acima de 60 anos correspondem a $77 \%$ dos cuidadores cônjuges.

As diferenças entre os graus de parentesco de relação cuidador-idoso mostraram que as categorias "outros" e "filhos" apresentaram maior benefício com a intervenção psicoeducacional para praticamente todas as variáveis. Entre os cuidadores "outros" não houve alteração nos escores entre antes e depois da intervenção apenas para as variáveis de satisfação geral com a vida e satisfação com a saúde. Os filhos tiveram aumento da satisfação geral com a vida, com a saúde, com a capacidade mental e com o envolvimento social, diminuição do escore na escala de depressão e aumento dos afetos positivos (em contrapartida, diminuição da satisfação com a capacidade física). Já os cuidadores cônjuges foram os que menos se beneficiaram da intervenção: apresentaram aumento somente da satisfação com o envolvimento social.

De acordo com a meta análise realizada por Sörensen et al. (2002), a dedicação e o impacto do cuidado diferenciam-se entre os diversos graus de parentesco. Como mostrado em alguns estudos analisados por esses autores, os cônjuges cuidadores sofrem mais para proverem o cuidado do que os filhos cuidadores, sugerindo que eles podem diferir em suas necessidades e em como se beneficiam da intervenção. Cuidadores filhos são mais propensos a perceber que entraram no papel de cuidador do que os cônjuges (Seltzer \& Wailing, 2000). Já que se espera que os filhos cuidem dos pais quando esses já não podem fazer por si só, o que pode ser menos frustrante para o filho aceitar esse novo papel e condição.

Outra questão que deve ser levada em conta é que cerca de $90 \%$ dos cuidadores cônjuges não dividiam a tarefa de cuidado com alguém, e, por outro lado, $80 \%$ dos cuidadores da categoria "outros" e 50\% dos cuidadores filhos recebiam ajuda para a realização das tarefas de cuidado. Esses dados podem ter influenciados os resultados do impacto da intervenção psicoeducacional sobre o bem-estar entre os diversos graus de parentesco.

Além disso, pode ser que cuidadores cônjuges se beneficiem menos de intervenções do que os filhos adultos, provavelmente, porque os filhos obtêm mais vantagens com a intervenção, pois muitas vezes são menos preparados para oferecer os cuidados do que os cônjuges. Cônjuges, possivelmente, já cuidaram de seus pais e desenvolveram estratégias de enfrentamento ou já reuniram informações importantes sobre os serviços e apoio da comunidade a partir de sua experiência anterior. A informação crucial que a intervenção psicoeducacional proporciona é mais novidade para os filhos e, portanto, mais eficaz na redução da sobrecarga e aumento do bem-estar (Sörensen et al., 2002). Outro ponto a considerar é que cônjuges relatam mais declínio nas atividades de lazer, piora nas relações familiares e menor satisfação matrimonial, provavelmente, porque a incapacidade do parceiro conjugal gera solidão e falta de reciprocidade (Seltzer \& Wailing, 2000).

Os valores das diferenças entre pré e pós-intervenção das variáveis de bem-estar analisadas entre os grupos de renda inferior a cinco salários mínimos e de renda superior a seis salários mínimos mostraram que os cuidadores com maior renda apresentaram menores níveis de bem-estar, e principalmente menor satisfação com a capacidade mental do que os cuidadores de baixa renda. Esses resultados sugerem que os cuidadores com menor renda são mais adaptados às condições adversas, e por isso apresentaram melhora em determinadas variáveis do bem-es- 
tar (satisfação com a capacidade mental, com o envolvimento social e aumento dos afetos positivos), já que, provavelmente, receberam informação e um suporte social que antes não tinham. E ainda, pode ser que cuidadores com maiores recursos financeiros sejam mais insatisfeitos com a situação em que vivem, porque, provavelmente, tinham altas expectativas para esse momento da vida, que não incluíam exercer o papel de cuidador. Além disso estar diante de um quadro neurodegenerativo sem prognóstico de cura, pode contribuir para que os cuidadores de alta renda sintam-se impotentes diante dessa situação, gerando maior desgaste emocional.

Um estudo realizado por Hansen, Slagsvold e Moum (2008), mostrou que idosos de baixa renda são mais satisfeitos porque usam estratégias adaptativas ou de acomodação. Devido às limitações e perdas endêmicas decorrentes do envelhecimento, à escassez de recursos econômicos, e às oportunidades limitadas para melhorar a sua situação econômica, as pessoas tendem a ajustar as suas necessidades, aspirações e utilizam padrões de comparação descendente à medida que envelhecem, a fim de manter o bem-estar. Embora tenha sido um estudo feito com idosos e não relataram se haviam cuidadores dentre esses, tal afirmação pode ser condizente com o presente estudo já que a maioria dos cuidadores era idosa (62\%).

Contudo, parece não haver consenso entre a associação positiva entre renda e satisfação com a vida na literartura gerontológica. Pinquart e Sörensen (2000) apontaram que idosos com maior renda relatam níveis mais altos de satisfação com a vida e felicidade. A variabilidade dessas correlações dependeu do gênero e da idade, ou seja, o bem estar subjetivo dos homens foi mais influenciado pela renda, enquanto que das mulheres foi pelo suporte social. Entre os idosos mais idosos, o bem-estar subjetivo teve correlação significativa com suporte social e entre os idosos mais jovens, a significância maior foi com renda. Drentea e Goldner (2006) verificaram maior frequência de sintomas depressivos em cuidadoras afro-americanas em comparação com cuidadoras brancas, explicado, em grande parte, porque as cuidadoras afro-americanas apresentam pior condição socioeconômica, maior demanda rela- cionada ao cuidado, pior condição física e por não serem casadas.

Em relação ao tempo de cuidado, observou-se que, os cuidadores que cuidavam há mais de doze meses foram os que se beneficiaram mais com a intervenção psicoeducacional. Eles tiveram aumento significativamente estatístico da satisfação geral com a vida quando comparados aos cuidadores que cuidavam há menos de doze meses $(p=0,036)$, aumento da satisfação com o envolvimento social e aumento dos afetos positivos após a intervenção. Enquanto que os cuidadores que cuidavam há menos tempo tiveram discreto aumento da satisfação com a capacidade mental e com o envolvimento pessoal.

Cuidadores que cuidam há mais tempo podem já estar mais adaptados com a tarefa de cuidar e estão mais conformados com a condição em que vivem. Esse resultado é condizente com o modelo de cuidado definido por Lawton, Moss, Hoffman e Perkinson (2000) que sugere que o impacto do cuidado muda ao longo do tempo, pois há um processo de adaptação à situação do cuidado, ou seja, o senso de ônus e estresse são altos no início, mas posteriormente diminuem, à medida que o cuidador torna-se mais competente para realizar as tarefas de cuidar e estabelece novos patamares de equilíbrio para si próprio. De acordo com Uwakwe (2006), o diagnóstico de demência pode ser desconcertante para o cuidador, dando origem a uma menor satisfação com a vida, diante das novas obrigações que exigem o cuidado. Após um longo período de realização das tarefas de cuidado, o cuidador pode desenvolver alguns mecanismos positivos de enfrentamento que possibilitam a adaptação a esse novo papel.

Embora os resultados referentes às variáveis de bem-estar estudadas não tenham sido estatisticamente significativos, sabe-se que os cuidadores podem beneficiar-se com a compreensão da natureza e das implicações da doença de seu familiar, mesmo não havendo impacto imediato sobre o bem-estar. De acordo com Zarit e Leitsch (2001), os cuidadores que primeiramente aprendem as implicações da doença de seu ente querido podem tornar-se mais deprimidos em curto prazo. No entanto, o entendimento é provavelmente um passo necessário para uma melhor 
adaptação em longo prazo. Da mesma forma, parece intrinsecamente benéfico para cuidadores receberem apoio e compreensão das pessoas ao seu redor, embora este apoio pode, necessariamente, não melhorar o bem-estar. Mesmo que uma intervenção não resulte em melhorias globais, pode ser julgado como um sucesso porque reuniu um conjunto de objetivos comuns aos que cuidam.

É importante considerar que cuidar não é uma tarefa linear, nem tampouco, simples. As flutuações frequentes - e até mesmo diárias - da condição do idoso com demência e o senso de sobrecarga associado ao cuidado, leva ao cuidador aos mais variados sentimentos positivos e negativos, intercalando momentos de equilíbrio e crise (Stephens, Franks, \& Townsend, 1994). A percepção de bem-estar e a avaliação que o cuidador faz da situação em que vive depende da fase de doença do idoso, das relações familiares, das circunstâncias envolvidas nos cuidar, da disponibilidade de recursos pessoais e externos, em diferentes momentos e situações e da história anterior de relacionamento entre idoso e familiar (Neri \& Sommerhalder, 2006). Tais fatores devem ser levados em conta para a interpretação dos resultados referentes ao impacto da intervenção psicoeducacional sobre o bem-estar de cuidadores com idosos com DA.

\section{Conclusão}

O grupo psicoeducacional apresentou um impacto positivo em relação às determinadas variáveis de bem-estar estudadas. Contudo, tais resultados se diferenciaram entre grupos de cuidadores, sugerindo que os cuidadores que cuidam há mais tempo têm maiores ganhos em relação à satisfação geral com a vida após a participação no grupo psicoeducacional, quando comparados aos cuidadores que cuidam há menos tempo, e cuidadores de menor renda, maior satisfação com a capacidade mental do que os cuidadores de renda acima de seis salários mínimos. Esse dado indica que a intervenção psicoeducacional proposta nesse estudo deve adequar-se às necessidades dos cuidadores de idosos que cuidam há menos de um ano, visto que a adaptação a esse novo papel de cuidador pode gerar maior sofri- mento emocional, considerando que esse é um período de transição em que os cuidadores são inevitavelmente confrontados com novas responsabilidades. Faz-se necessário também considerar as expectativas dos cuidadores de maior renda, a fim de contribuir para o aumento do bem-estar desse grupo.

\section{Referências}

Abreu, I. D., Barros, H. L., \& Forlenza, O. V. (2005). Demência de Alzheimer: Correlação entre memória e autonomia. Revista de Psiquiatria Clínica, 32(3), 131-136.

Aldwin, C. M., Yancura, L. A., \& Boeninger, D. K. (2007). Coping, health, and aging. In C. M. Aldwin, C. L. Park, \& A. Spiro (Eds.), Handbook of health psychology and aging (pp. 185-198). New York: The Guilford Press.

Almeida, O. P., \& Almeida, S. A. (1999). Short versions of the Geriatric Depression Scale: A study of their validity for the diagnosis of major depressive episode according to ICD-10 and DSMIV. International Journal of Geriatric Psychiatry, 14(10), 858-865.

Andrade, A. M. E. (2009). Escuela de Cuidadores: como programa psicoeducativo para cuidadores informales de adultos mayores con demencia. Revista Cubana de Salud Pública, 35(2).

Aneshensel, C. S., Pearlin, L. I., Mulan, J. T., Zarit, S. H., \& Whitlach, C. J. (1995). Profiles of caregiving. The unexpected career. San Diego, CA: Academic Press.

Arritxabal, I., Soler, A. G., Silva, A. I., Artola, E. U., Gozález, I. L., Veiga, P. D., \& Lezaun, J. J. (2011). Efectos del entrenamiento en estrategias de regulación emocional en el bienestar de cuidadores de enfermos de Alzheimer. Revista Española de Geriatría y Gerontología, 46, 206212.

Bookwala, J. (2009). The impact of parent care on marital quality and well-being in adult daughters and sons. Journal of Gerontology: Psychological Sciences, 64B(3), 339-347.

Bookwala, J., \& Schulz, R. (2000). A comparison of primary stressors, secondary stressors, and depressive symptoms between elderly caregiving husbands andwives: The Caregiver Health Effects Study. Psychology and Aging, 15, 607-616. 
Brown, N. (2011). Psychoeducational Groups: Process and practice ( $3^{\text {rd }}$ ed.). New York: Routledge.

Chien, L. Y., Chu, H., Guo, J. L., Liao, Y. M., Chang L. I., Chen, C. H., \& Chou, K. R. (2011, February). Caregiver support groups in patients with dementia: A meta-analysis. International Journal Geriatric Psychiatry, 9. doi: 10.1002/ gps. 2660

Dahlberg, L., Demack, S., \& Bambra, C. (2007, September). Age and gender of informal carers: A population-based study in the UK. Health Social Care Community, 15(5), 439-445.

Drentea, P., \& Goldner, M. A. (2006). Caregiving outside of the home: The effects of race on depression. Ethnicity and Health, 11, 41-57.

Ducharme, F., Levesque, L., Giroux, F., \& Lachance, L. (2005). Follow-up of an intervention program for caregivers of a relative with dementia living in a long-term care setting: Are there any persistent and delayed effects? Aging \& Mental Health, 9(5), 461-469.

Ducharme, F., Levesque, L., Lachance, L., Kergoat, M. J., \& Coulombe, R. (2011). Challenges associated with transition to caregiver role following diagnostic disclosure of Alzheimer disease: A descriptive study. International Journal of Nursing Studies. doi: 10.1016/j.ijnurstu. 2011.02.011

Figueiredo, A. L., Souza, L., Dell'Áglio, J. C., Jr., \& Argimon, I. I. L. (2009). O uso da psicoeducação no tratamento de transtorno bipolar. Revista Brasileira de Terapia Comportamental Cognitiva, 11(1), 15-24.

Fortinsky, R. H., Tennen, H., Frank, N., \& Affleck, G. (2007). Health and psychological consequences of caregiving. In C. M. Aldwin, C. L. Park, \& A. Spiro (Eds.), Handbook of health psychology and aging (pp. 210-226). New York: The Guilford Press.

Gallagher-Thompson, D., \& DeVries, H. (1994). Coping with frustration classes: development and preliminary outcomes with women who care for relatives. Gerontologist, 34, 548-552.

Gottlieb, B. H., Thompson, L. W., \& Bourgeois, M. (2003). Monitoring and evaluating interventions. In D.W. Coon, D. Gallagher-Thompson, \& L. W. Thompson, Innovative interventions to reduce dementia caregiver distress (pp. 28-49). New York: Springer.

Haley, W. (1997). The family caregiver's role in Alzheimer's disease. Neurology, 48(5), 25-29.
Hansen, T., Slagsvold, B., \& Moum, T. (2008). Financial satisfaction in old age: A satisfaction paradox or a result of accumulated wealth? Social Indicators Research, 89(2), 323-347.

Hosaka, T., \& Sugiyama, Y. (2003). Structured intervention in family caregivers of the demented elderly and changes in their immune function. Psychiatry and Clinical Neurosciences, 57(2), 147-151.

Lavoie, J. P., Ducharme, F., Levesque, L., Hebert, R., Vezina, J., Gendron, C., Voyer, ...L. (2005). Understanding the outcomes of a psycho-educational group intervention for caregivers of persons with dementia living at home: A process evaluation. Aging Mental Health, 9(1), 25-34.

Lawton, M. P., Moss, M., Hoffman, C., \& Perkinson, M. (2000). Two transitions in daughters' caregiving careers. The Gerontologist, 40, 437-448.

Lemos, N. D., Gazzola, J. M., \& Ramos, L. R. (2006). Cuidando do paciente com Alzheimer: O impacto da doença no cuidador. Saúde e Sociedade, 15(3), 170-179.

Luzardo, A. R., Gorini, M. I. P. C., \& Silva, A. P. S. S. (2006). Características de idosos com Doença de Alzheimer e seus cuidadores: Uma série de casos em um serviço de neurogeriatria. Texto Contexto Enfermagem, 15(4), 587-594.

Neri, A. L. (2002). Bienestar subjetivo en la vida adulta y en la vejez: hacia una psicologia positiva en la América Latina. Revista Latinoamericana de Psicologia, 34, 55-74.

Neri, A. L. (2010). Desafios ao bem-estar físico e psicológico enfrentados por idosos cuidadores no contexto da família: Dados do FIBRA Campinas. In A. A. Camarano (Org.), Cuidados de longa duração para a população idosa: Um novo risco social a ser assumido? (pp. 303-336). Rio de Janeiro, RJ: Fundação Instituto de Pesquisa Econômica Aplicada.

Neri, A. L., \& Sommerhalder, C. (2006). As várias faces do cuidador e do bem-estar do cuidador. In A. L. Neri (Org.), Cuidar do idoso no contexto da família: Questões psicológicas e sociais (pp. 9-64). Campinas, SP: Alínea.

Pimenta, G. M. F., Costa, M. A. S. M. C., Gonçalves, L. H. T., \& Alvarez, A. M. (2009). Perfil do familiar cuidador de idoso fragilizado em convivio doméstico da grande região do Porto, Portugal. Revista Escola de Enfermagem da USP, 43(3), 609-614. 
Pinquart, M., \& Sörensen, S. (2000). Influences of socioeconomic status, social network, andcompetence on subjective well-being in later life: A meta-analysis. Psychology and Aging, 15, 187224.

Pinquart, M., \& Sörensen, S. (2003). Differences between caregivers and noncaregivers in Psychological Health and Physical Health: A metaanalysis. Psychology and Aging, 18(2), 250-267.

Pinquart, M., \& Sörensen, S. (2006a). Helping caregivers of persons with dementia: Which interventions work and how large are their effects? International Psychogeriatrics, 18, 577-595. doi: $10.1017 / \mathrm{S} 1041610206003462$

Pinquart, M., \& Sörensen, S. (2006b). Gender differences in caregiver stressors, social resources, and health: An updated meta-analysis. The Journal of Gerontology: Psychological Sciences, 61B(1), 33-45.

Seltzer, M. M., \& Waiting, L. (2000). The dynamics of caregiving: Transitions during a three-year prospective study. The Gerontologist, 40(2), $165-178$.

Sheikh, J. I., \& Yesavage, J. A. (1986). Geriatric Depression Scale (GDS). Recent evidence and development of a shorter version. In T. L. Brink, Clinical gerontology: A guide to assessment and intervention (pp. 165-173). New York: The Haworth Press.

Siqueira, M. M. M., Martins, M. C. F., \& Moura, O. I. (1999). Construção e validação fatorial da EAPN: Escala de ânimo positivo e negativo. Revista da Sociedade de Psicologia do Triângulo Mineiro, 2(3), 34-40.
Sörensen, S., Pinquart, M., \& Duberstein, P. (2002). How effective are interventions with caregivers? An updated meta-analysis. Gerontologist, 42(3), 356-372.

Stephens, M. A., Franks, M. M., \& Townsend, A. L. (1994). Stress and reward in women`s multiple roles: The case of women in the middle. Psychology and Aging, 9, 45-52.

Uwakwe, R. (2006). Satisfaction with dementia care - Giving in Nigeria: A pilot investigation. International Journal of Geriatric Psychiatry, 21, 296-297.

Winefield, H. R., \& Harvey, E. J. (2004). Needs of family caregivers in chronic schizophrenia. Schizophrenia Bull, 20(3), 557-566.

World Health Organization, \& Alzheimer`s Disease International. (2012). Dementia: A public health priority. Geneva, Switzerland: Department of Mental Health and Substance Abuse, World Health Organization.

Zarit, S. H., \& Leitsch, S. A. (2001). Developing and evaluating community based intervention programs for Alzheimer's patients and their caregivers. Aging \& Mental Health, 5(1), S84-S98.
Recebido: 04/07/2012

$1^{a}$ revisão: $22 / 11 / 2012$

$3^{a}$ revisão: 06/12/2012

Aceite final: 11/12/201 